\title{
A floating inferior alveolar neurovascular bundle in a simple bone cyst: A case report
}

\author{
SHINPEI MATSUDA, HITOSHI YOSHIMURA and KAZUO SANO \\ Department of Dentistry and Oral Surgery, Unit of Sensory and Locomotor Medicine, \\ Division of Medicine, Faculty of Medical Sciences, University of Fukui, Yoshida, Fukui 910-1193, Japan
}

Received July 22, 2018; Accepted February 28, 2019

DOI: $10.3892 /$ etm.2019.7463

\begin{abstract}
Simple bone cysts (SBCs) are uncommon lesions that usually occur in the long bones of the skeleton and in the body of the mandible. Displacement of the mandibular canal due to cystic lesions have been reported previously. However, there have been no reports in which endoscopy has been utilized to observe an inferior alveolar neurovascular bundle in the lesion lumen. This current study presents a case of mandibular SBC accompanied by a floating inferior alveolar neurovascular bundle. A 13-year-old boy presented to the Department of Dentistry and Oral Surgery, University of Fukui in March 2017 with a painless swelling in the right buccal region. He was treated with fenestration surgery using the tooth extraction socket of the right mandibular third molar. An endoscopic view of the lesion lumen presented a surrounding cortical bone without an epithelial lining and the floating inferior alveolar neurovascular bundle that was consistent with the linear low signal area of the preoperative T2-weighted magnetic resonance imaging. SBC often develops in young people, and as such is minimal surgical invasion is preferable. The current case indicated that clinicians should consider the possibility of the floating inferior alveolar neurovascular bundle in the lesion and should not damage the neurovascular bundle in the narrow surgical field.
\end{abstract}

\section{Introduction}

Simple bone cysts (SBC) are fluid-containing cystic lesions that are surrounded by a hard bony wall without an epithelial lining and without evidence of infection (1). The etiology of SBC is still controversial (2). Despite possessing many

Correspondence to: Dr Shinpei Matsuda, Department of Dentistry and Oral Surgery, Unit of Sensory and Locomotor Medicine, Division of Medicine, Faculty of Medical Sciences, University of Fukui, 23-3 Matsuokashimoaizuki, Eiheiji-cho, Yoshida, Fukui 910-1193, Japan

E-mail: shinpeim@u-fukui.ac.jp

Key words: inferior alveolar neurovascular bundle, simple bone cyst, endoscope alternate names, ncluding solitary, traumatic, hemorrhagic or unicameral bone cysts, the classification of bone tumors and associated lesions included in the fourth edition of the World Health Organization's classification of head and neck tumors recommends using the term simple bone cyst (3). SBC represents $\sim 1 \%$ of all jaw cysts (4). In the second decade of life, SBC usually occurs in the metaphyseal region of long bones and in the body of the mandible $(2,4,5)$. In addition, SBC that forms in the maxillomandibular region has no sex predilection $(2,4)$. Although mandibular SBC is usually asymptomatic and detected incidentally by radiographs, it may cause swelling due to bone expansion, pain, pathologic fractures and hypoesthesia of the inferior alveolar nerve $(2,4,6-8)$. In general, SBC has a well-delineated unilocular radiolucent lesion and a scalloped appearance at the upper edge extending between the roots $(4,5)$. Displacement of the mandibular canal as a result of mandibular cystic lesions, including SBC, has also been reported $(6,8,9)$. The majority of these reports detected mandibular canal displacement via panoramic radiograph and computed tomography (CT) scanning. However, no reports have described the detection of the inferior alveolar arteriovenous nerve bundle in the lesion lumen using an endoscope. The current study reports a case of mandibular SBC accompanied by a floating inferior alveolar neurovascular bundle that was diagnosed using an endoscope.

\section{Case report}

A 13-year-old boy presented to the Department of Dentistry and Oral Surgery at the University of Fukui Hospital (Fukui, Japan) in March 2017 with a painless swelling in the right buccal region that had been present for the last 1.5 months. Physical examination revealed facial asymmetry due to swelling in the right buccal region. Intraoral examination uncovered a hard diffuse bony bulging of the right posterior mandible. The patient had no remarkable medical history or history of trauma.

Panoramic radiograph and enhanced computed tomography (CT) scanning revealed a well-delineated unilocular radiolucent lesion ranging from the first premolar to the mandibular ramus with lamina dura resorption accompanied with bony expansion. This radiolucent lesion had a maximum anteroposterior and buccolingual measurement of 70x30 mm. The mandibular canal could not be confirmed by panoramic 
radiograph and enhanced $\mathrm{CT}$ examinations of the lesion area (Figs. 1 and 2). In the lesion area, magnetic resonance imaging (MRI) demonstrated homogeneous intermediate signal intensity on the T1-weighted image and homogeneous high signal intensity including linear low signal area, which was considered an inferior alveolar nerve on the T2-weighted image (Fig. 3A and B). Laboratory experiments were conducted at the Department of Clinical Laboratory in University of Fukui Hospital to find the white blood cell count $(6,100$ cells $/ \mu 1$; normal range, 3,300-8,600 cells $/ \mu \mathrm{l})$ and $\mathrm{C}$-reactive protein level $(0.01 \mathrm{mg} / \mathrm{dl}$; normal range, $0-0.14 \mathrm{mg} / \mathrm{dl})$; no abnormal findings were revealed. The normal ranges were based on the result of measurements performed by the Department of Clinical Laboratory in University of Fukui Hospital. The results of these examinations indicated that the lesion was SBC or odontogenic cyst. To minimize the surgical invasion, the authors planned to treat the lesion with fenestration surgery using the tooth extraction socket of the right mandibular third molar.

A total of 2 weeks following the first visit, the authors performed the fenestration surgery under general anesthesia. The alveolar bone of the right mandibular third molar region was removed and the serous fluid was released as soon as the instrument reached the lesion. The impacted right mandibular third molar was then extracted. No neoplastic lesion or cyst wall was found in the lesion by visual observation from the tooth extraction socket of the right mandibular third molar. An endoscope was subsequently inserted through the socket and the lesion lumen was observed, confirming that the lesion lumen was surrounded by cortical bone without an epithelial lining. These results indicated that the lesion was SBC. Furthermore, an inferior alveolar neurovascular bundle floating in the lesion lumen was confirmed via endoscopic examination (Fig. 4). Endoscopy also revealed that the inferior alveolar neurovascular bundle branched off into the incisive and mental nerve bundle in the anterior part of the lesion lumen. These results were consistent with the linear low signal area of the preoperative T2-weighted MRI. The tooth extraction socket was packed with gauzes including Bacitracin-fradiomycin sulfate (TOYO Pharmaceutical Co., Ltd, Osaka, Japan). Cefazolin sodium (2 g/day, twice a day) was administered intravenously for 5 days, and then Cefdinir (300 mg/day, three times a day; both Astellas Pharma Inc., Tokyo, Japan) was orally administered for 4 days.

Histopathological examinations performed by another scientist in the division of Surgical Pathology, University of Fukui (Fukui, Japan). The examinations of the soft tissues around the right third molar exhibited no evidence of neoplasia. The patient was subsequently diagnosed with SBC based on the results of histopathological examination, transparent serous content fluid from the lesion and intraoperative results including those of endoscopy.

The postoperative course, such as postoperative haemorrhage or postoperative pain, was uneventful and swelling in the right buccal region decreased. The tooth extraction socket of the right mandibular third molar was closed 4 months following the first visit. A follow-up CT scan was performed 5 months following the first visit (Fig. 5). It revealed bone regeneration of the lesion area and the regeneration of the mandibular canal in the linear low signal area of the T2-weighted preoperative MRI

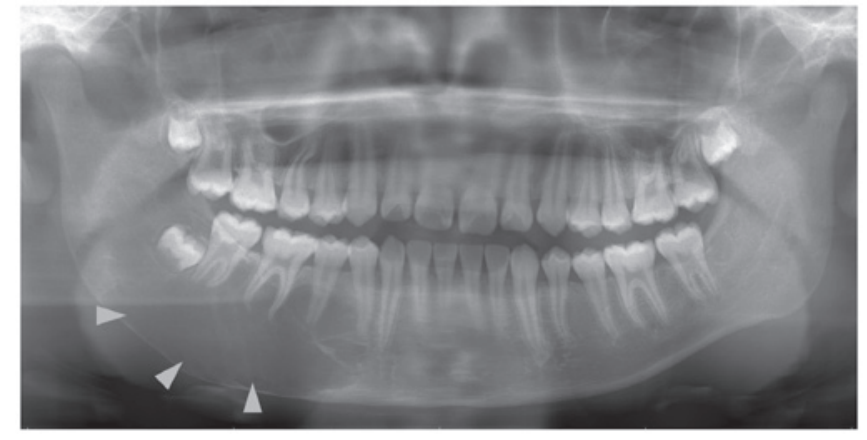

Figure 1. A panoramic radiograph obtained at the patient's first visit reveals a radiolucent lesion accompanied with a bony expansion in the right mandibular body and ramus (as indicated by the grey triangles).

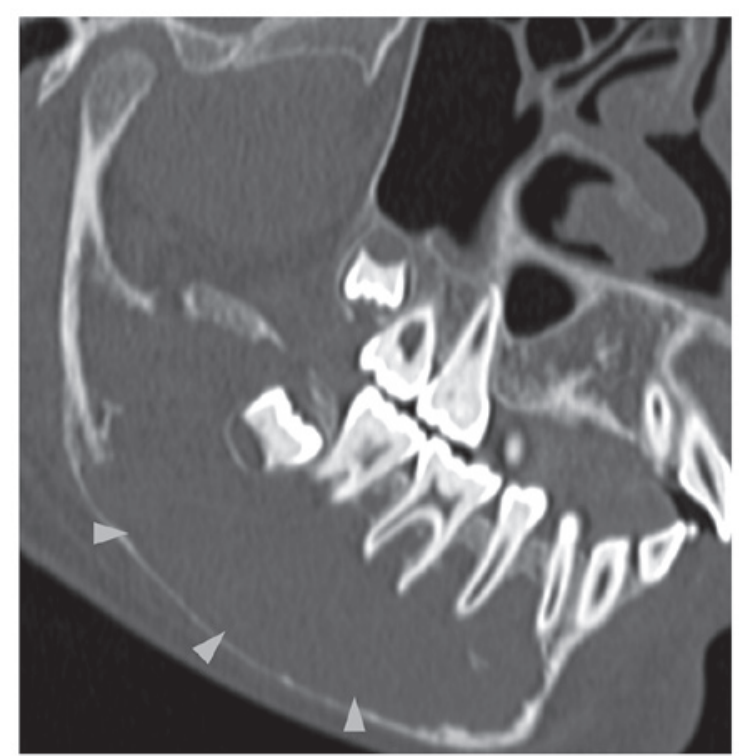

Figure 2. A computed tomography scan at the patient's first visit revealed a unilocular lesion with a well-defined margin and lamina dura resorption (as indicated by the grey triangles); the mandibular canal could not be confirmed in the lesion area.

was confirmed. A total of 17 months after the operation, clinical findings associated with recurrence have not been observed.

\section{Discussion}

In the present case, an inferior alveolar neurovascular bundle floating in the lesion lumen of SBC was confirmed endoscopically. These results were consistent with the linear low signal area of the preoperative T2-weighted MRI. Furthermore, regeneration of the mandibular canal in the linear low signal area of the T2-weighted preoperative MRI was confirmed in the postoperative follow-up CT scan 5 months following the first visit.

The etiology of SBC is controversial, but three predominant hypotheses have been presented for its occurrence: i) An abnormality of bone growth, ii) the tumor degeneration process and iii) traumatic-hemorrhagic theory (4). Although the traumatic-hemorrhagic theory is the most widely accepted, $>50 \%$ of patients with SBC have no history of trauma (4). Further, the patient of the present case exhibited no remarkable 

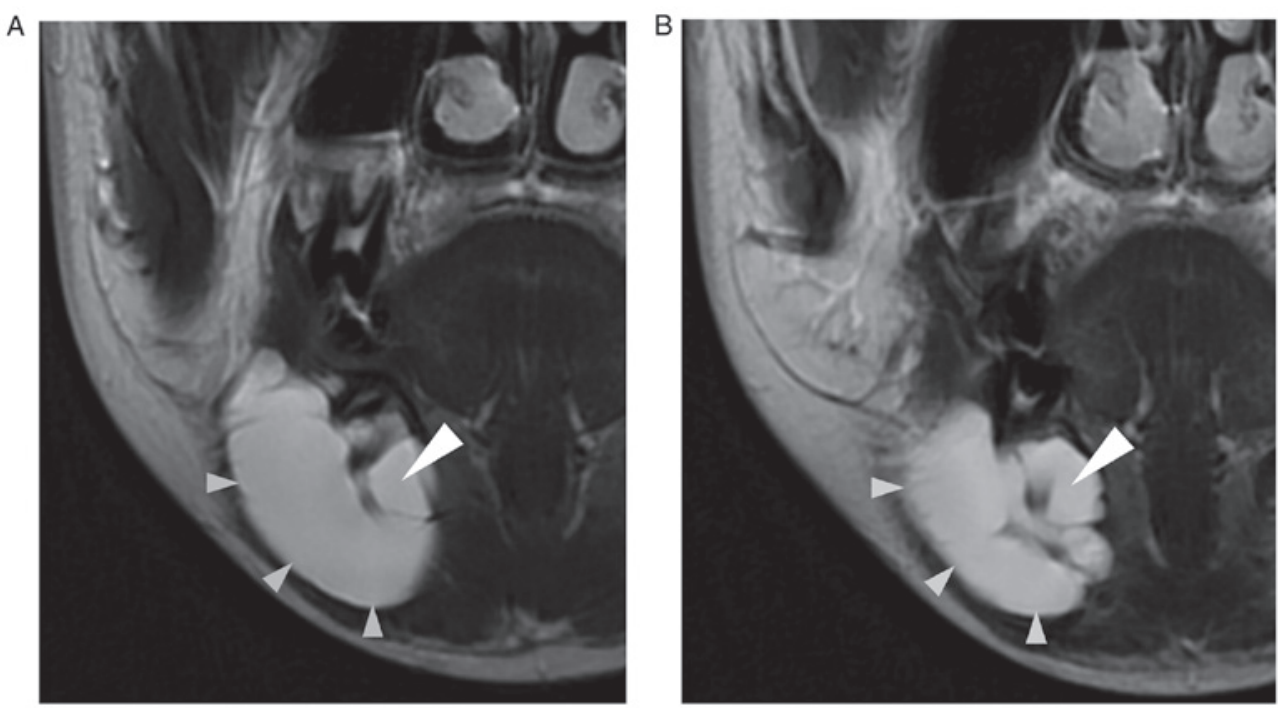

Figure 3. (A) Magnetic resonance imaging at the patient's first visit exhibiting a homogeneous high signal intensity including a linear low signal area, which was considered an inferior alveolar nerve, on a T2-weighted image of the lesion area. (B) Magnetic resonance imaging at the patients' first visit exhibiting the inferior alveolar neurovascular bundle branched off into the incisive and mental nerve bundle in the anterior part of the lesion lumen. The inferior alveolar nerve is indicated by the white triangle and the lesion area is indicated by the grey triangles.

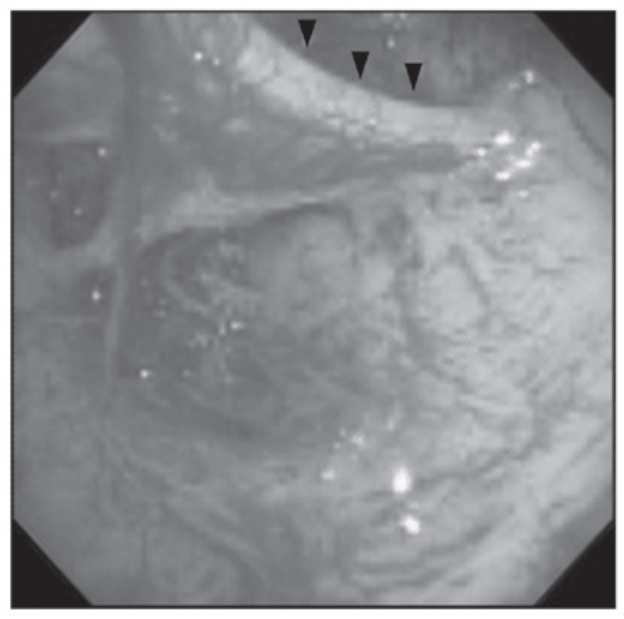

Figure 4. An endoscopic image of the lesion lumen presenting the cortical bone without an epithelial lining. The inferior alveolar neurovascular bundle (as indicated by black triangles) branches off into the incisive and mental nerve bundle.

traumatic history. Harnet et al (4) have indicated that the traumatic-hemorrhagic theory may apply to the mandible due to microtraumas to the teeth and alveolar bone. Previous literature has also reported cases of SBC accompanied by an expansion of the cortical plate and the displacement of the mandibular canal $(6,8,9)$. Mathew et al $(8)$ speculated that the displacement of the mandibular canal is caused by a local rise in osmotic pressure or intraosseous hematoma formation, which may support the traumatic-hemorrhagic theory as etiology. In addition, Matsumura et al (6) analyzed 53 SBCs and reported that there was no significant association between the radiographic margin, tooth margins, displacement of the mandibular canal, and histopathological results.

Hatakeyama et al (2) reported a case of mandibular condyle SBC treated using an endoscope, which had the advantage of observing the lesion lumen of the mandible with

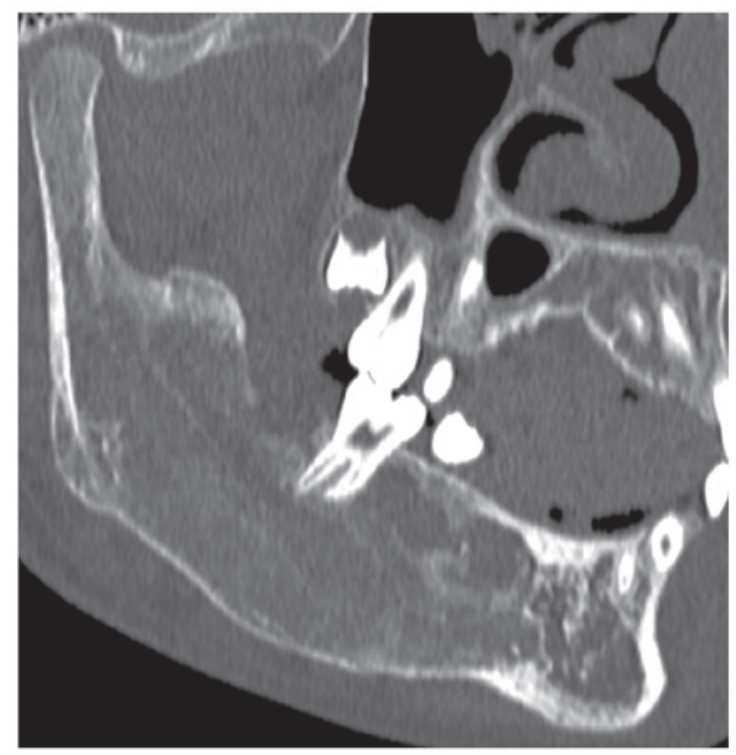

Figure 5. A follow-up computed tomography scan 5 months following the patients' first visit presenting bone regeneration of the lesion area and the mandibular canal.

minimal surgical invasion. In the current case, an endoscope was inserted through the tooth extraction socket of the right mandibular third molar, from which the entire lesion lumen was observed. Consequently, it was confirmed that the lesion lumen was surrounded by cortical bone without an epithelial lining, which is a characteristic of SBCs. The present case indicated that the combination of tooth extraction and endoscopy minimizes surgical invasion for the treatment of SBC including the third molar tooth.

Surgical exploration and biopsy are necessary to diagnose various radiolucent lesions, including SBC $(10,11)$. Treatment methods for SBC include curettage of the bone wall, fenestration, packing the cavity, aspiration and osteotomy (12). 
Curettage is often sufficient to stimulate bleeding and facilitate osteogenesis (11). Additionally, graft materials are used for treatment of larger SBCs (10). However, SBC often heals spontaneously (11). To minimize surgical invasion, fenestration surgery using the tooth extraction socket of the right mandibular third molar was performed in the present case. Suei et al (12) reported that recurrence was observed in 2 of 13 patients with SBC that were treated using fenestration. In addition, Suei et al suggested an association between the prognosis and radiographic features of SBCs, including absent lamina dura, scalloped margins, nodular bone expansion, radiopaque mass and multiple cavities (12). In the present case, the postoperative course was uneventful and a follow-up CT scan 5 months after the first visit revealed bone regeneration in the lesion area. However, the follow-ups will continue for the foreseeable future as a long-term follow-up is necessary to account for the possibility of recurrence, as the radiographic examination performed at the first visit revealed resorbed lamina dura.

Several limitations of endoscopic surgical procedure for SBCs should be considered. First, it may be difficult to use in SBCs with a multilocular appearance included internal structure, including trabeculae or septa (13). Second, some SBCs do not exhibit bony expansions (14). Third, SBCs with teeth, which are acceptable for dental extraction in lesion areas, are rare $(13,14)$.

In conclusion, the present case revealed that inferior alveolar neurovascular bundles can float through the lesion lumen of SBC. Furthermore, T2-weighted MRI may be useful in confirming the course of the inferior alveolar neurovascular bundle in SBC. As SBC is often observed in the second decade of life (2), it is preferable to minimize surgical invasion. However, in the narrow surgical field, clinicians should consider the possibility of the floating inferior alveolar neurovascular bundle in the lesion and avoid damage to the neurovascular bundle itself.

\section{Acknowledgements}

Not applicable.

\section{Funding}

No funding was received.

\section{Availability of data and materials}

All data generated or analyzed during this study are included in this published article.

\section{Authors' contributions}

SM contributed to the conception and writing the manuscript. HY and KS revised the manuscript. All authors wrote the original medical record, analyzed the clinical data, and read and approved the final version of the manuscript.

\section{Ethics approval and consent to participate}

Not applicable.

\section{Patient consent for publication}

Written informed consent was obtained from the patient's parents for the publication of the case and accompanying images.

\section{Competing interests}

The authors declare that they have no competing interests.

\section{References}

1. Rushton MA: Solitary bone cysts in the mandible. Br Dent J 81: 37-49, 1946.

2. Hatakeyama D, Tamaoki N, Iida K, Yonemoto K, Kato K, Makita H, Toida M and Shibata S: Simple bone cyst of the mandibular condyle in a child: Report of a case. J Oral Maxillofac Surg 70: 2118-2123, 2012.

3. Speight PM and Takata T: New tumour entities in the 4th edition of the World Health Organization Classification of Head and Neck tumours: Odontogenic and maxillofacial bone tumours. Virchows Arch 472: 331-339, 2018.

4. Harnet JC, Lombardi T, Klewansky P, Rieger J, Tempe MH and Clavert JM: Solitary bone cyst of the jaws: A review of the etiopathogenic hypotheses. J Oral Maxillofac Surg 66: 2345-2348, 2008.

5. Suomalainen A, Apajalahti S, Kuhlefelt M and Hagström J: Simple bone cyst: A radiological dilemma. Dentomaxillofac Radiol 38: 174-177, 2009.

6. Matsumura S, Murakami S, Kakimoto N, Furukawa S, Kishino M, Ishida T and Fuchihata H: Histopathologic and radiographic findings of the simple bone cyst. Oral Surg Oral Med Oral Pathol Oral Radiol Endod 85: 619-625, 1998.

7. Strabbing EM, Gortzak RA, Vinke JG, Saridin CP and van Merkesteyn JP: An atypical presentation of a solitary bone cyst of the mandibular ramus: A case report. J Craniomaxillofac Surg 39: 145-147, 2011.

8. Mathew R, Omami G, Gianoli D and Lurie A: Unusual cone-beam computerized tomography presentation of traumatic (simple) bone cyst: Case report and radiographic analysis. Oral Surg Oral Med Oral Pathol Oral Radiol 113: 410-413, 2012.

9. MacDonald-Jankowski DS: Traumatic bone cysts in the jaws of a Hong Kong Chinese population. Clin Radiol 50: 787-791, 1995.

10. Robinson RA and Vincent SD: Idiopathic and developmental abnormalities. In: AFIP atlas of tumor pathology series 4, Tumor and cysts of the jaws. ARP Press, Silver Spring, Maryland, pp. 43-48, 2012.

11. El-Naggar AK, Chan JKC, Grandis JR, Takata T and Slootweg PJ (ed.): Giant cell lesions and bone cysts. In: World Health Organization classification of tumors, WHO classification of head and neck tumours. WHO Press, Geneva, pp. 256-260, 2017.

12. Suei Y, Taguchi A, Nagasaki T and Tanimoto K: Radiographic findings and prognosis of simple bone cysts of the jaws. Dentomaxillofac Radiol 39: 65-71, 2010.

13. Imanimoghaddam M, Javadian Langaroody A, Nemati S and Ataei Azimi S: Simple bone cyst of the mandible: Report of two cases. Iran J Radiol 8: 43-46, 2011.

14. Madiraju G, Yallamraju S, Rajendran V and SrinivasaRao K: Solitary bone cyst of the mandible: A case report and brief review of literature. BMJ Case Rep 2014: bcr2013200945, 2014. 